\title{
ÁREAS PRIORITARIAS DE CONSERVACIÓN PARA LA FLORA LEÑOSA DEL ESTADO DE COLIMA, MÉXICO
}

\author{
Juan MartíneZ-CruZZ ${ }^{1,2}$ y Guillermo IbarRa-ManRíQuez ${ }^{1}$ \\ ${ }^{1}$ Universidad Nacional Autónoma de México, Centro de Investigaciones en \\ Ecosistemas, Antigua carretera a Pátzcuaro 8701, Colonia Ex Hacienda de San José \\ de la Huerta, 58190 Morelia, Michoacán, México. \\ 22Autor para la correspondencia: jmc@oikos.unam.mx
}

\section{RESUMEN}

En el estado de Colima se realizó un ejercicio sobre las prioridades de conservación de su flora leñosa (árboles y lianas), usando postulados de los métodos sistemáticos de la planeación de la conservación (gradisectos, grupos indicadores y cuatro métodos iterativos para ponderar la riqueza total, el valor de rareza de las especies, así como el número y abundancia de las endémicas de México). Con base en la geomorfología, altitud y tipos de vegetación en el área de estudio se delimitaron 53 unidades ambientales (UA). Para la estratificación del muestreo se establecieron cuatro gradisectos que contienen una representación de $91.6 \%$ de las UA del estado. Dentro de ellos se eligieron las 15 con mayor superficie y con vías de comunicación accesibles para ubicar 24 sitios de muestreo de 0.1 ha, donde todos los árboles y lianas con d.a.p. $\geq 2.5 \mathrm{~cm}$ fueron determinados. Se registraron 4419 individuos que incluyen 417 especies y 71 familias. Los sitios contrastaron marcadamente en su riqueza de especies (entre 82 y 5), la que se correlacionó de manera significativa con la de las endémicas a México $(r=0.85$, $\mathrm{P}<0.001$ ). Para conservar al menos $75 \%$ de las especies, cinco sitios se ubicaron siempre entre las seis primeras posiciones en los cuatro métodos iterativos utilizados. Al incluir en este ejercicio a los taxones registrados en dos reservas (la Reserva de la Biosfera Manantlán y el Área de Protección Forestal y Refugio de la Fauna Silvestre El Jabalí), se observa que desempeñan un papel muy relevante, ya que permiten la protección de $67.4 \%$ del total de especies y $72.5 \%$ de las endémicas. Cuatro sitios resultaron ser los más complementarios respecto a los componentes florísticos de las reservas. Dado que las UA donde se establecen los mismos no están conectadas, se sugiere que las tres unidades más cercanas se enlacen por medio de corredores de vegetación, los cuales podrían extenderse hacia la porción sureña de la reserva de Manantlán. Se recomienda considerar en propuestas futuras de conservación algunos tipos de vegetación valiosos por su diversidad en otras formas de crecimiento (e. g. 
el componente herbáceo de los bosques de Pinus-Quercus) o por los servicios ecosistémicos que aportan (e. g. manglares). Se enfatiza la necesidad de generar información similar a la que presenta este estudio para otros grupos de plantas y animales para establecer un sistema más representativo de las áreas de conservación en el estado.

Palabras clave: árboles, especies endémicas, gradisectos, lianas, métodos iterativos, riqueza, unidades ambientales.

\begin{abstract}
The diversity of the woody flora (trees and lianas) of the state of Colima was quantified and prioritized using principles of systematic conservation planning (gradsects, indicators groups and four heuristic algorithms for weighting the species richness, the rarity value, and besides the number and density of species endemic to Mexico). A total of 53 environmental units (EU) were defined with information of geomorphology, altitude and vegetation types. Four gradsects embraced most of the EU diversity (91.6\%). Within gradsects 15 EU were chosen with the largest area and accessible roads to locate 24 sampling sites of 0.1 ha, where all trees and lianas $\geq 2.5 \mathrm{~cm}$ dap were identified. We counted 4419 individuals, including 417 species and 71 families. Species richness among sites contrasted markedly (between 82 and 5 species). Site species richness significantly correlated with the species endemic to Mexico $(r=0.85, \mathrm{P}<0.001)$. To preserve at least $75 \%$ of the species, five sites were always selected in the top six using the four iterative methods. When this analysis was repeated considering the species recorded in two reserves (Biosphere Reserve of Manantlán and Forest Protection and Wildlife Refuge Area El Jabalí), both areas played a key role, allowing the protection of $67.4 \%$ of the total species and $72.5 \%$ of the endemics. Four sites were the most complementary if compared with the flora of the reserves. Since the EU where these sites are located are not connected, we suggest that three nearby units be linked through a system of vegetation corridors, which could expand toward the southern portion of the Manantlán reserve. It is recommended that future conservation proposals consider types of vegetation that are valued for possesing other growth forms (e. g., the herbaceous understory of Pinus-Quercus forest) or for the ecosystematic functions they provide (e. g., mangroves). To reach the goal of a representative network of biodiversity areas in Colima, similar data for other groups of plants and animals must be generated.
\end{abstract}

Key words: endemic species, environmental units, gradsects, heuristic algorithms, lianas, species richness, trees. 
Martínez-Cruz e Ibarra-Manríquez: Áreas prioritarias de conservación para la flora leñosa de Colima

\section{INTRODUCCIÓN}

En la práctica, la planificación de áreas de conservación de la biodiversidad se ve afectada, en ocasiones de manera drástica, por restricciones de tipo económico, social y político (Margules y Pressey, 2000). A pesar de lo anterior, estos autores indican que los criterios biológicos relevantes para la conservación podrían tener mayor influencia al respecto si se dispone de una estrategia que asegure su representatividad regional, para lo cual proponen un esquema con fases de desarrollo explícitas. Una de las etapas fundamentales dentro del mismo es la compilación de datos para ser usados como sustitutos de la diversidad biológica (e. g. subconjuntos de especies o distintos tipos de habitats), dentro de la cual los inventarios biológicos juegan un papel primordial.

Sin embargo, los recursos económicos, el personal capacitado y el tiempo requerido para llevar a cabo inventarios biológicos para seleccionar áreas prioritarias de conservación son limitados (Austin y Heyligers, 1991; Raven y Wilson, 1992; Stork, 1994), por lo que éstas tradicionalmente han sido establecidas tomando en cuenta especies carismáticas con diferentes grados de amenaza, sitios con poco o nulo interés económico para el ser humano y la naturalidad regional (Margules y Usher, 1981; Pressey et al., 1994). Debido a lo anterior, no siempre es posible conservar sitios biológicamente importantes por su riqueza o grado de endemismo, lo que disminuye las posibilidades de contar con sistemas representativos de áreas de protección (Margules et al., 1988; Caldecott et al., 1996; Gaston, 1996; Margules y Pressey, 2000; Pressey y Taffs, 2001, pero ver Ceballos, 2007).

Una alternativa para contar con adecuados inventarios biológicos es el uso de transectos dirigidos a gradientes o gradisectos (gradsects), ya que en ellos se combina un trabajo de campo intensivo y de bajo costo para inferir en el menor tiempo posible la diversidad biológica presente en un área particular (Austin y Heyligers, 1991; Gillison, 1983; Margules y Redhead, 1995; Wessels et al., 1998). Los gradisectos incluyen el uso de grupos indicadores y algoritmos para priorizar áreas de conservación (métodos iterativos). Los primeros se han usado para inferir la riqueza de organismos más problemáticos de inventariar debido, entre otras razones, a su pequeño tamaño o a su desconocimiento taxonómico (Di Castri et al., 1992; Halffter y Favila, 1993; Gaston, 1996; Margules y Pressey, 2000; Jayasuriya y Abayawardana, 2008). El uso de estos grupos para ubicar áreas de conservación cuenta con una literatura amplia y entre los taxones que se han empleado al respecto están: I) artrópodos (arañas e insectos; Virolainen et al., 2000; Cardoso et al., 2004; Finch y Löffler, 2010), II) hongos (Basidiomicetes; Virolainen et al., 2000), III) mariposas 
(Papilionidae; Fleishman et al., 2005), IV) plantas (árboles; Villaseñor et al., 2003; Schmit et al., 2005) y V) vertebrados (peces, anfibios, reptiles, aves y mamíferos; Fleishman et al., 2005).

Con respecto a los métodos iterativos, éstos priorizan sitios para la conservación de la biodiversidad con base en la importancia relativa del número de especies, evaluando su grado de complementaridad y qué tan particulares son con respecto a los taxones que albergan, además que permiten considerar las restricciones que puedan tener ciertas áreas para ser conservadas y ser flexible en cuanto a las opciones que pueden aportar, lo que incrementa su eficiencia y representatividad (Margules et al., 1994, 2002; Margules y Pressey, 2000; Ceballos, 2007).

La porción occidental de México presenta áreas con escaso conocimiento biológico, a pesar de formar parte de Mesoamérica, una de las regiones más importantes a nivel mundial por la biodiversidad que alberga (Myers et al., 2000; Olson y Dinerstein, 2002). El estado de Colima se ubica en el occidente mexicano (Fig. 1A) y en su pequeño territorio se presenta un gradiente altitudinal muy amplio, que va desde el nivel del mar hasta los $4225 \mathrm{~m}$, lo que facilita la confluencia de diferentes tipos de vegetación tropical y templada (Rzedowski y McVaugh, 1966). Si bien el conocimiento florístico de Colima fue considerado deficiente por Dávila y Sosa (1994), la situación ha empezado a mejorar paulatinamente (Cuevas et al., 1998, Padilla-Velarde et al., 2006, 2008) y es sin duda un estado con una biodiversidad importante. Una muestra de lo anterior es que Padilla-Velarde y colaboradores (2006) enlistan 550 especies de árboles, de los cuales $43.4 \%$ son endémicos a México y tres restringen su área de distribución a Colima. Lamentablemente, esta entidad no está exenta de cambios drásticos del área ocupada por su vegetación natural, ya que para el año 2000 se había reducido en 47.6\% (Palacio-Prieto et al., 2000), además de que sólo $360 \mathrm{~km}^{2}$ están considerados bajo alguna categoría de protección (Flores-Villela y Gerez, 1994).

Tomando en cuenta la variedad de tipos de vegetación que alberga, la diversidad y particularidad de su flora, el grado de amenaza que se cierne sobre ella debido a los cambios de uso de suelo y la escasez de áreas de protección estatales, Colima es un candidato relevante para realizar un ejercicio sobre prioridades de conservación. En consecuencia, el presente trabajo pretende contestar como preguntas principales: I) ¿el método de gradisectos es eficiente en el muestreo de áreas con alta diversidad florística como lo es el estado de Colima?, II) ¿dónde se encuentran los sitios prioritarios de conservación para la flora leñosa (árboles y lianas) de este estado?, III) ¿qué grado de coincidencia existe entre los sitios con mayor riqueza de especies y los más relevantes con respecto al endemismo? y IV) ¿cuál es la contribución de las reservas del estado en la conservación de esta flora? 


\section{MATERIALES Y MÉTODOS}

El estado de Colima se localiza en la parte oeste de México (Fig. 1A), entre los $18^{\circ} 41^{\prime}-19^{\circ} 31^{\prime} \mathrm{N}$ y $103^{\circ} 29^{\prime}-104^{\circ} 41^{\prime}$ O (Anónimo, 2001), limitando hacia el oriente con los ríos Coahuayana y Tamazula, mientras que la frontera noroccidental la constituye el río Cihuatlán (Anónimo, 1981). En el territorio colimense confluyen las provincias terrestres Sierra Madre del Sur y Eje Neovolcánico, con altitudes entre 0 y 4225 m (Anónimo, 2001). Estos trabajos indican la presencia de dos provincias hidrográficas: Costa de Jalisco y Armería-Coahuayana; dentro de ellas destacan los ríos Cihuatlán y Purificación, así como el arroyo de Agua Zarca. En cuanto al clima, cinco tipos diferentes convergen en Colima, siendo el más importante el cálido subhúmedo $(\mathrm{A}(\mathrm{w}))$ que abarca $77.8 \%$ del estado, seguido en orden de importancia por los siguientes (García, 1981): semiseco (BS $)$, semicálido (A(c)), templado (C) y semifrío (C (E)). Respecto a su geología superficial, en este estado se encuentran formaciones metamórficas que datan desde el Jurásico hasta depósitos aluviales del Cuaternario (Anónimo, 1981), sobre los que se desarrollan distintas variantes húmicas, dístricas, háplicas, pélicas o calcáricas de suelos como litosoles, feozem, rendzinas, regosoles, leptosoles y vertisoles, e incluso se registran en la zona costera los de categoría gleysol y solonchak (Anónimo, 1981, 1983). Los tipos de vegetación donde los árboles son elementos predominantes son (Palacio-Prieto et al., 2000): bosque de encino, bosque de pino, bosque de pino-encino, bosque mesófilo de montaña, bosque de galería, manglar, palmar, sabana, selva baja espinosa, así como variantes caducifolias y subcaducifolias de la selva baja y de la selva mediana. Descripciones más detalladas al respecto pueden encontrarse en Rzedowski y McVaugh (1966).

Se utilizó el sistema de información geográfica ILWIS ${ }^{\circledR}$ (Integrated Land and Water Information System) para delimitar categorías geomorfológicas, reclasificar tipos de vegetación y delimitar unidades ambientales (UA). Las categorías geomorfológicas fueron obtenidas a través de un levantamiento geomorfológico sintético (Verstappen y Van Zuidam, 1991), mediante el cual a partir del modelo digital de elevación 1:250,000, se realizó la interpretación de formas de relieve, basándose en los procesos geomorfológicos, la geoforma, la pendiente y la altitud (Cuadro 1). Posteriormente, se hizo un levantamiento en el terreno (verificación) y se elaboró un mapa en formato raster, con 11 categorías geomorfológicas. La extensión de los tipos de vegetación se obtuvo a partir del Inventario Nacional Forestal (Palacio-Prieto et al., 2000), utilizando sólo las que sin duda contaban con árboles como elementos fisonómicos notables. Para la elaboración del mapa de unidades ambientales se realizó una sobreposición de la información de las categorías geomorfológicas y las de 


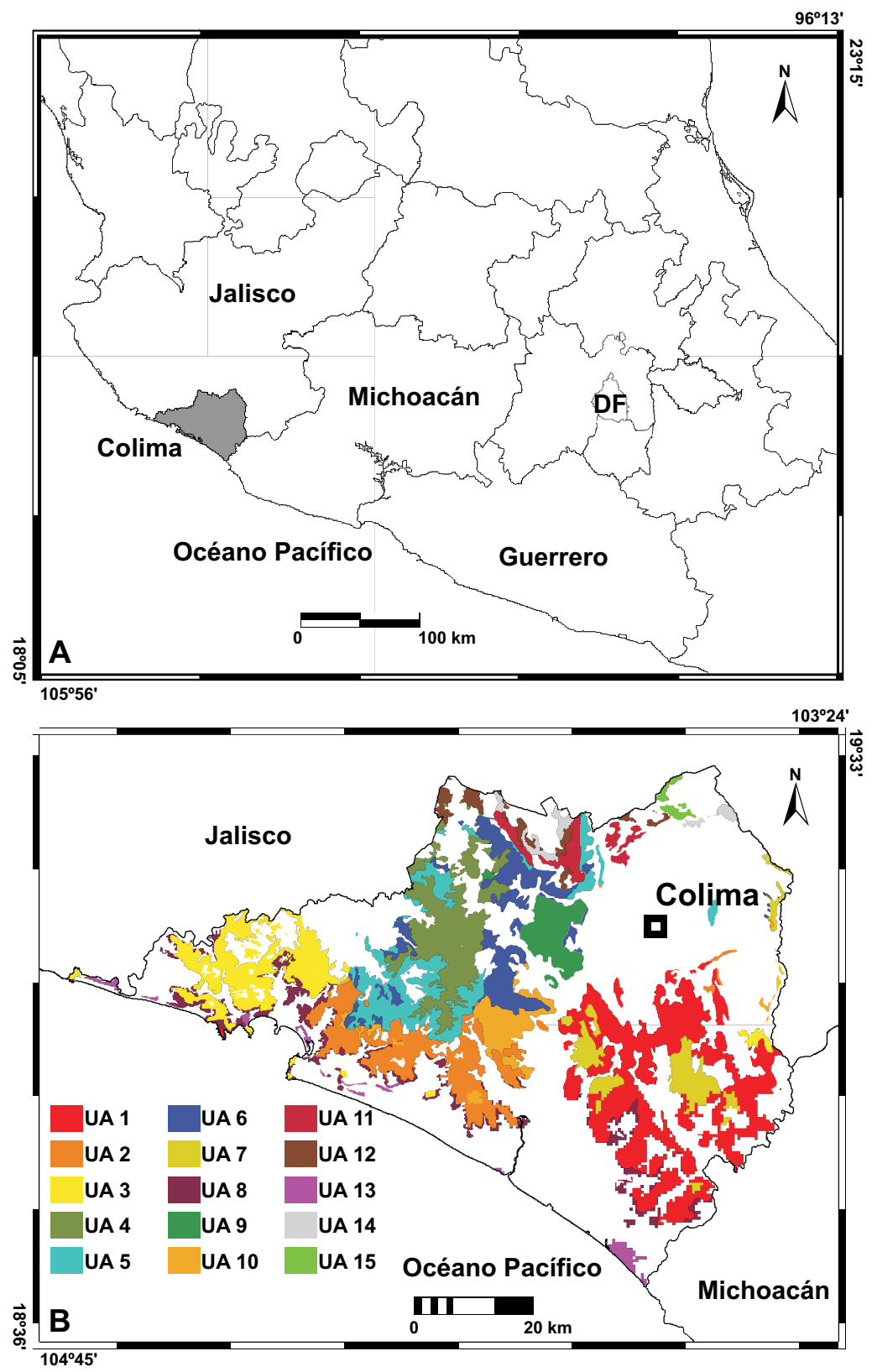

Fig. 1. A) Ubicación geográfica del estado de Colima. B) Distribución de las 15 unidades ambientales donde se establecieron los sitios de muestreo de la vegetación. Las áreas de color blanco son las categorías de uso de suelo no considerado en los análisis (p.e. vegetación acuática). 
Martínez-Cruz e Ibarra-Manríquez: Áreas prioritarias de conservación para la flora leñosa de Colima

Cuadro 1. Atributos de geoforma, pendiente y altitud, los cuales sirvieron de base para obtener las categorías geomorfológicas de Colima.

\begin{tabular}{clll}
\hline $\begin{array}{c}\text { Categoría } \\
\text { geomorfológica }\end{array}$ & \multicolumn{1}{c}{ Geoforma $\left(\mathrm{km}^{2}\right)$} & Pendiente & \multicolumn{1}{c}{$\begin{array}{c}\text { Altitud } \\
\text { (m s.n.m. })\end{array}$} \\
\hline 1 & Planicie baja (1051.6) & menor de $6^{\circ}$ & $0-100$ \\
2 & Planicie alta (173.2) & menor de 6 & mayor de 100 \\
3 & Meseta baja (573.1) & $3-6^{\circ}$ & $500-1000$ \\
4 & Meseta alta (45.9) & $3-6^{\circ}$ & mayor de 1000 \\
5 & Piedemonte inferior (641.1) & $3-15^{\circ}$ & $250-500$ \\
6 & Piedemonte medio (1110.2) & $3-15^{\circ}$ & $500-1000$ \\
7 & Piedemonte superior (426.2) & $3-15^{\circ}$ & $1000-1500$ \\
8 & Lomeríos bajos (344.9) & $6-20^{\circ}$ & $250-500$ \\
9 & Lomeríos altos (960.5) & $6-20^{\circ}$ & $500-1500$ \\
10 & Montaña baja (145.2) & mayor de 20 & $1000-2000$ \\
11 & Montaña alta (84.5) & mayor de 20 & mayor de 2000 \\
\hline
\end{tabular}

vegetación, con lo que se obtuvo una carta, en formato raster (pixel de $100 \times 100 \mathrm{~m}$ ), con 53 UA. Este mapa puede ser obtenido solicitándolo al primer autor.

Uno de los supuestos fundamentales en los gradisectos es que dentro de sus límites se debe representar la mayor heterogeneidad ambiental posible (Gillison, 1983), que en el presente estudio se traduce en la incorporación del mayor número posible de UA, combinando la cantidad, la orientación y el tamaño (largo $\times$ ancho) de los gradisectos. En este punto no existe una propuesta concreta, ya que ello depende de la heterogeneidad ambiental del área a inventariar. En la zona de estudio se hicieron varias combinaciones de los gradisectos, dirigiéndolos principalmente a lo largo del gradiente altitudinal del estado y tratando de incluir la mayoría de las UA (Fig. 2A). Para decidir si cuatro gradisectos eran representativos de las 53 UA definidas en Colima, se decidió que el porcentaje del área de las UA en Colima y en los gradisectos no debía exceder de $\pm 10 \%$ (Cuadro 2). Esta proporción de variación fue el mínimo que pudo cumplirse para todas las UA y en la figura 1B se muestran las 15 con mayor cobertura en el estado.

Para el adecuado muestreo de las UA se integraron al análisis dos consideraciones que permitieran optimizar el tiempo, el costo económico y el personal humano necesario para el muestreo. La primera de ellas consiste en ubicar los sitios de 


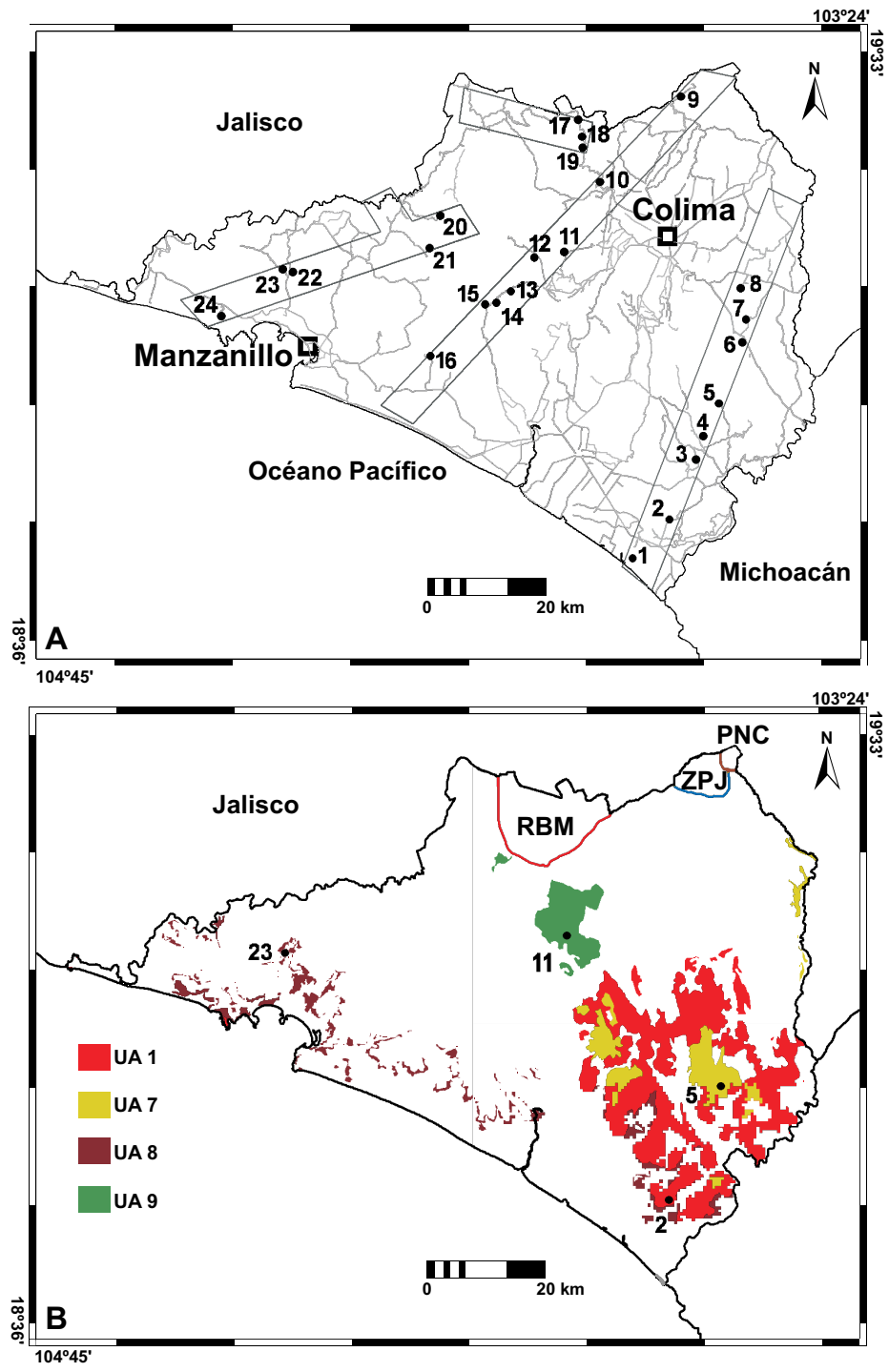

Fig. 2. A) Localización de los cuatro gradisectos usados en el presente estudio, indicando dentro de éstos los sitios de muestreo. También se muestra la red de caminos estatales (líneas grises). B) Ubicación de los sitios con mayor grado de coincidencia en los diferentes algoritmos usados (sitios 2, 5, 11 y 23) y distribución en Colima de las unidades ambientales (UA) donde éstos se encuentran. Los límites de los polígonos en Colima de las reservas naturales se señalan en color: rojo para la Reserva de la Biosfera Manantlán (RBM), azul pálido para el Área de Protección Forestal y Refugio de la Fauna Silvestre El Jabalí (ZPJ) y en color pardo el Parque Nacional Nevado de Colima (PNC). 
muestreo a una distancia no mayor de un kilómetro de alguna vía de comunicación (Margules y Redhead, 1995; Margules y Pressey, 2000). Para lograr este objetivo, la red de caminos de Colima (Anónimo, 1985) fue añadida como una capa adicional

Cuadro 2. Unidades ambientales en la cuales se ubicaron los 24 sitios de muestreo en Colima, arregladas con base en el tamaño de su área. Para cada unidad se indica el porcentaje en área que presenta en el estado, en los gradisectos y la diferencia entre ambos valores, el cual no debería sobrepasar $\pm 10 \%$.

\begin{tabular}{|c|c|c|c|c|c|}
\hline \multicolumn{2}{|c|}{ Unidades Ambientales } & \multirow{2}{*}{$\begin{array}{c}\text { Sitios } \\
2,3,4, \\
6,7\end{array}$} & \multirow{2}{*}{$\begin{array}{c}\begin{array}{c}\text { Área } \\
\text { Colima }(\%)\end{array} \\
10.03\end{array}$} & \multirow{2}{*}{$\begin{array}{c}\begin{array}{c}\text { Área } \\
\text { gradisectos }(\%)\end{array} \\
13.02\end{array}$} & \multirow{2}{*}{$\begin{array}{c}\begin{array}{c}\text { Diferencia entre } \\
\text { ambas áreas }\end{array} \\
2.99\end{array}$} \\
\hline 1) & $\begin{array}{l}\text { Lomeríos altos-selva baja sub } \\
\text { y caducifolia }\end{array}$ & & & & \\
\hline 2) & $\begin{array}{l}\text { Piedemonte inferior-selva } \\
\text { baja sub y caducifolia }\end{array}$ & 8,16 & 4.57 & 5.04 & 0.47 \\
\hline 3) & $\begin{array}{l}\text { Lomeríos bajos-selva baja } \\
\text { sub y caducifolia }\end{array}$ & 24,22 & 4.55 & 12.74 & 8.19 \\
\hline 4) & $\begin{array}{l}\text { Piedemonte medio-bosque de } \\
\text { encino }\end{array}$ & 20,21 & 4.55 & 6.59 & 2.04 \\
\hline 5) & $\begin{array}{l}\text { Piedemonte medio-selva baja } \\
\text { sub y caducifolia }\end{array}$ & 15,10 & 4.18 & 6.13 & 1.95 \\
\hline 6) & $\begin{array}{l}\text { Piedemonte medio-selva } \\
\text { mediana sub y caducifolia }\end{array}$ & 12,13 & 3.67 & 8.96 & 5.29 \\
\hline 7) & $\begin{array}{l}\text { Lomeríos altos-selva mediana } \\
\text { sub y caducifolia }\end{array}$ & 5 & 2.40 & 6.13 & 3.73 \\
\hline 8) & $\begin{array}{l}\text { Planicie baja-selva baja sub y } \\
\text { caducifolia }\end{array}$ & 23 & 2.34 & 4.01 & 1.67 \\
\hline 9) & $\begin{array}{l}\text { Montaña baja-selva mediana } \\
\text { sub y caducifolia }\end{array}$ & 11 & 1.82 & 7.35 & 5.53 \\
\hline 10) & $\begin{array}{l}\text { Piedemonte inferior-selva } \\
\text { mediana sub y caducifolia }\end{array}$ & 14 & 1.82 & 2.32 & 0.5 \\
\hline 11) & $\begin{array}{l}\text { Piedemonte superior-selva } \\
\text { baja sub y caducifolia }\end{array}$ & 19 & 1.15 & 3.49 & 2.34 \\
\hline 12) & $\begin{array}{l}\text { Piedemonte superior-bosque } \\
\text { de encino }\end{array}$ & 18 & 0.86 & 3.13 & 2.27 \\
\hline 13) & Planicie baja-manglar & 1 & 0.62 & 2.21 & 1.59 \\
\hline 14) & $\begin{array}{l}\text { Piedemonte superior-bosque } \\
\text { pino-encino }\end{array}$ & 17 & 0.61 & 1.39 & 0.78 \\
\hline 15) & $\begin{array}{l}\text { Montaña alta-bosque mesófilo } \\
\text { de montaña }\end{array}$ & 9 & 0.27 & 1.30 & 1.03 \\
\hline
\end{tabular}


al mapa de las UA y de los gradisectos (Fig. 2A); las 15 UA elegidas previamente por su similar representatividad en Colima y en los gradisectos cumplieron también esta premisa (Figs. 1B, 2A). La segunda condición fue realizar un mayor esfuerzo de muestreo (5 sitios) en la UA con más superficie y en la medida que su área porcentual disminuyó, el muestreo se acotó a uno o dos sitios (Cuadro 2).

El método de Gentry (1982), ampliamente usado en el muestreo de comunidades tropicales, se empleó para censar la vegetación en los 24 sitios seleccionados, el cual consiste en 10 líneas de $50 \times 2 \mathrm{~m}(0.1 \mathrm{ha})$, que se disponen de manera paralela entre sí respecto a la pendiente, con una distancia de separación de $25 \mathrm{~m}$. Dentro de cada transecto se midieron e identificaron todos los árboles enraizados con un diámetro a la altura del pecho (d.a.p.) $>2.5 \mathrm{~cm}$, incluyendo a las lianas arraigadas en los transectos que presentaron este diámetro en la base de su tallo. Se recolectaron ejemplares botánicos para su posterior identificación, la cual se realizó por medio de la revisión de floras regionales y ejemplares depositados en los herbarios IEB (Herbario del Centro Regional del Bajío del Instituto de Ecología, A.C.) y MEXU (Herbario Nacional del Instituto de Biología, Universidad Nacional Autónoma de México) o por consulta directa a taxónomos.

Para la selección de las áreas prioritarias se utilizaron los datos de los muestreos realizados y en un análisis independiente se añadieron las especies leñosas registradas en la Reserva de la Biósfera Sierra Manantlán (Cuevas et al., 1998) y en el Área de Protección Forestal y Refugio de la Fauna Silvestre El Jabalí (Rothschild et al., 1992); para el caso de la primera se eliminaron las plantas registradas exclusivamente para el estado de Jalisco. De aquí en adelante ambas reservas sólo se mencionarán como Manantlán y El Jabalí, respectivamente.

El primero de los protocolos utilizados se basa en la riqueza de especies obtenidas en el muestreo. Este método se sustenta en la propuesta de Villaseñor et al. (2003), pero se modificó en el presente estudio en lo referente al porcentaje de especies con interés para su conservación (de 100\% a sólo 75\%). El algoritmo consiste de los siguientes pasos: 1) elegir el sitio que contenga la mayor riqueza de especies, las cuales ya no son consideradas en las fases subsecuentes del análisis; a las no eliminadas se les denomina el complemento, 2) se selecciona el lugar que contribuya con el mayor número de especies de este último, 3) cuando dos o más sitios contengan el mismo complemento, se elige el que incluya el mayor número de especies únicas al sitio, 4) en caso de que persista el empate, se escoge el que se encuentre más cercano a alguno de los seleccionados previamente, y 5) tal ejercicio se continúa hasta que $75 \%$ de las especies hayan sido incorporadas. Este mismo algoritmo se ejecutó con las plantas de distribución restringida a México que se obtuvieron en el muestreo; tal condición se 
asignó para los taxones determinados hasta especie con base en Padilla-Velarde et al. (2006), mientras que para las lianas fue necesario consultar diversos trabajos florísticos (e. g. Rzedowski y Calderón de Rzedowski, 1993). Los ejercicios de selección con el total de especies y con el contingente endémico fueron repetidos añadiendo al análisis las especies de la flora leñosa de las reservas de Manantlán y El Jabalí.

Otro protocolo utilizado se basa en el valor de endemismo (VE), desarrollado por Kerr (1997) y modificado por Villaseñor y colaboradores (2003). En el presente trabajo se denominará como valor de rareza VR y se calcula con la siguiente fórmula:

$$
V R=\sum_{i=1}^{S} Q^{-1}
$$

donde $\mathrm{S}$ es la cantidad total de especies registradas en los muestreos y $Q$ es el número de sitios ocupados por cada una de ellas. El algoritmo en este trabajo se obtiene de la manera siguiente: 1) se calcula el inverso de la frecuencia de los sitios donde se ha registrado cada especie en el área de estudio, 2) se calcula el VR por sitio, el cual se obtiene de la suma del inverso de las frecuencias de las presentes en cada sitio particular, 3) el lugar con mayor prioridad de conservación será el que tenga el monto más alto de VR, 4) las especies del sitio elegido se eliminan de las fases subsecuentes del análisis, 5) hecha esta exclusión, se debe recalcular el VR de cada sitio y el elegido para ser conservado será otra vez el que tenga el mayor de este algoritmo, 6) en caso de que dos o más sitios tengan un coeficiente similar, se elige aquel que tenga la mayor riqueza de taxones endémicos, 7) si persiste el empate, se elige el más cercano a alguno de los previamente seleccionados y 8) el procedimiento termina cuando se ha logrado la inclusión de $75 \%$ de las especies. Este algoritmo fue calculado también con el contingente florístico de los árboles y lianas de las reservas de Manantlán y El Jabalí. Finalmente, el último ejercicio consiste en la selección de los sitios con base en el número de individuos de las especies endémicas que albergan, es decir, priorizándolos de acuerdo con los valores más altos de abundancia de los taxones restringidos a México; el ejercicio finaliza cuando se incluye al menos $75 \%$ de los individuos.

\section{RESULTADOS}

En los 24 sitios muestreados ( 2.4 ha) se registraron 4419 plantas con un d.a.p. $\geq$ $2.5 \mathrm{~cm}$, que representan un total de 417 especies (355 arbóreas y 62 lianas), agrupadas 
en 200 géneros y 71 familias, que incluyen un total de 76 especies endémicas de México (75 arbóreas y una liana). El grado de determinación taxonómica fue de 297 a nivel de especie (256 fueron árboles y 41 lianas), 37 a género, 75 a familia y 8 morfoespecies. $\mathrm{El}$ sitio con mayor riqueza fue el número 11 (UA 9 montaña baja-selva mediana sub y caducifolia) con 82 especies (Cuadro 3), mientras que el valor más bajo (5 especies) se obtuvo en el sitio 1 (UA 13 planicie baja-manglar). El sitio 5 (UA 7 lomeríos altos-selva mediana sub y caducifolia) presentó la mayor riqueza de elementos endémicos con 22 especies, superando sólo con una al 11; el sitio 1 fue el único en carecer de taxones de distribución restringida (Cuadro 3). De acuerdo con el coeficiente de correlación de Pearson, el número de especies de los sitios se vincula de manera positiva y significativa con el que presentan las endémicas $(\mathrm{r}=0.85, \mathrm{P}<0.001)$.

Con respecto a los protocolos de conservación utilizados, la priorización basada en la riqueza de especies requiere de 10 sitios para incluir bajo algún esquema de conservación a 75\% de las leñosas (Cuadro 4). El sitio 11 ocupa el primer lugar, ya que contribuye con cerca de $20 \%$ de la diversidad total. El 5 adiciona 54 especies a las ya protegidas con la opción anterior, logrando con ello abarcar $32.7 \%$ de los taxones. Ocho sitios adicionan paulatinamente elementos hasta alcanzar el objetivo con el 12 (77.9\%). El análisis con la riqueza de especies endémicas indica que se requieren seis sitios para lograr conservar $80.2 \%$ de éstas, si bien la secuencia de prioridad obtenida es diferente al ejercicio de riqueza (Cuadro 4). En este caso, el sitio 5 es el más importante, ya que comprende $30.2 \%$ de los endemismos y al añadir el 23 se resguardaría $46 \%$ del componente de distribución restringida. De los seis sitios seleccionados con las endémicas, únicamente el 2 no figura en los priorizados por el algoritmo de riqueza total.

El valor de rareza seleccionó 12 sitios para proteger $76.2 \%$ de las especies registradas (Cuadro 4) y nuevamente hay coincidencias de selección con los algoritmos previos, ya que se comparten las localidades más importantes (5 y 9). La 9 resultó la primera prioridad de conservación, con $11.1 \%$ de las especies. El último ejercicio basado en la abundancia de los elementos endémicos indica que son necesarios 12 sitios para incluir a 75\% de los individuos (Cuadro 4). Entre los cinco más importantes al respecto está el 6 (168 individuos) y le siguen en prioridad el 2 (129), el 19 (121), el 10 (119) y el 11 (114); éstos contienen 37.4\% de los elementos endémicos. Tomando en cuenta las prioridades de los cuatro algoritmos, los sitios 5, 9, 11, 19 y 23 se ubican dentro de las seis primeras en al menos tres de los procedimientos usados.

Con excepción del ejercicio de priorización basado en la abundancia de especies endémicas, los demás fueron repetidos añadiendo las de árboles y lianas (255) 
de las áreas naturales protegidas de Colima (Cuadro 5), lo que en conjunto con las registradas en las 24 parcelas de muestreo determinadas hasta especie (297) dan un total de 552. El ejercicio de riqueza señala que Manantlán es la de mayor importancia, ya que comprende cerca de la mitad (53.4\%) de todas las especies leñosas

Cuadro 3. Número de especies (total y endémicas a México) y su respectiva abundancia por sitio para las lianas y árboles de Colima.

\begin{tabular}{ccccc}
\hline Sitio & $\begin{array}{c}\text { Total de } \\
\text { especies }\end{array}$ & $\begin{array}{c}\text { Número de } \\
\text { individuos }\end{array}$ & $\begin{array}{c}\text { Especies } \\
\text { endémicas }\end{array}$ & $\begin{array}{c}\text { Número de } \\
\text { individuos }\end{array}$ \\
\hline 1 & 5 & 84 & 0 & 0 \\
2 & 55 & 233 & 20 & 129 \\
3 & 43 & 220 & 15 & 104 \\
4 & 60 & 170 & 18 & 53 \\
5 & 76 & 193 & 22 & 88 \\
6 & 43 & 242 & 19 & 168 \\
7 & 26 & 176 & 12 & 103 \\
8 & 39 & 181 & 17 & 81 \\
9 & 35 & 62 & 6 & 18 \\
10 & 70 & 279 & 17 & 119 \\
11 & 82 & 322 & 21 & 114 \\
12 & 66 & 139 & 17 & 40 \\
13 & 67 & 237 & 14 & 57 \\
14 & 61 & 191 & 14 & 48 \\
15 & 76 & 225 & 16 & 47 \\
16 & 57 & 217 & 14 & 70 \\
17 & 20 & 91 & 4 & 9 \\
18 & 25 & 65 & 6 & 15 \\
19 & 47 & 232 & 14 & 121 \\
20 & 7 & 71 & 3 & 51 \\
21 & 7 & 87 & 3 & 80 \\
22 & 68 & 234 & 17 & 89 \\
23 & 67 & 247 & 19 & 108 \\
24 & 60 & 221 & 10 & \\
\hline
\end{tabular}


Cuadro 4. Prioridades de conservación para la flora leñosa del estado de Colima con base en cuatro algoritmos de selección. En cada prioridad se indica el sitio de muestreo seleccionado, el número de especies que conserva (total y especies endémicas), el valor de rareza y el número de individuos (densidad) de las especies endémicas. El porcentaje del total acumulado por sitio para los distintos algoritmos se muestra entre paréntesis. Los sitios coincidentes en las seis primeras posiciones y en al menos tres de los ejercicios utilizados se señalan en negritas.

\begin{tabular}{|c|c|c|c|c|c|c|c|c|}
\hline \multirow{2}{*}{ Prioridad } & \multicolumn{2}{|c|}{ Riqueza total } & \multicolumn{2}{|c|}{ Riqueza endémicas } & \multicolumn{2}{|c|}{ Valor de rareza } & \multicolumn{2}{|c|}{ Densidad de endémicas } \\
\hline & Sitio & $\begin{array}{l}\text { Especies } \\
(\mathrm{N}=417)\end{array}$ & Sitio & $\begin{array}{l}\text { Especies } \\
(\mathrm{N}=76)\end{array}$ & Sitio & $\begin{array}{l}\text { Especies } \\
(\mathrm{N}=417)\end{array}$ & Sitio & $\begin{array}{l}\text { Núm. individuos } \\
\qquad(\mathrm{N}=1737)\end{array}$ \\
\hline 1 & 11 & $82(19.7)$ & 5 & $23(30.2)$ & 9 & $29.6(11.1)$ & 6 & $168(9.6)$ \\
\hline 2 & 5 & $54(32.7)$ & 23 & $12(46.0)$ & 11 & $26.4(21.0)$ & 2 & $129(17.0)$ \\
\hline 3 & 15 & $37(41.6)$ & 19 & $8(56.5)$ & 5 & $22(29.3)$ & 19 & $121(24.0)$ \\
\hline 4 & 9 & $29(48.6)$ & 2 & $8(67.1)$ & 24 & $18.4(36.2)$ & 10 & $119(30.9)$ \\
\hline 5 & 23 & $26(54.8)$ & 9 & $5(73.6)$ & 19 & $17.8(42.9)$ & 11 & $114(37.4)$ \\
\hline 6 & 19 & $25(60.8)$ & 15 & $5(80.2)$ & 13 & $15.7(48.8)$ & 23 & $108(43.6)$ \\
\hline 7 & 13 & $21(65.9)$ & 6 & $4(85.5)$ & 16 & $14.8(54.4)$ & 3 & $104(49.6)$ \\
\hline 8 & 24 & $19(70.4)$ & 10 & $3(89.4)$ & 12 & $12.9(59.2)$ & 7 & $103(55.6)$ \\
\hline 9 & 16 & $17(74.5)$ & 12 & $2(92.1)$ & 23 & $12.4(63.9)$ & 22 & $89(60.7)$ \\
\hline 10 & 12 & 14 (77.9) & 18 & $1(93.4)$ & 4 & $11.3(68.1)$ & 5 & $88(65.8)$ \\
\hline 11 & 18 & $13(81.0)$ & 14 & $1(94.7)$ & 17 & $10.8(72.2)$ & 8 & $81(70.4)$ \\
\hline 12 & 4 & $12(83.9)$ & 8 & $1(96.0)$ & 22 & $10.5(76.2)$ & 21 & $80(75.0)$ \\
\hline
\end{tabular}

(Cuadro 5); los territorios de ambas reservas protegen 67.4\% (372) de todos los taxones. Posterior a esta selección, se requieren dos sitios para alcanzar el objetivo de proteger $75 \%$ de las especies. Cuando se implementa el ejercicio de conservación, ahora con 120 endémicas a México (76 obtenidas en los sitios de muestreo y 44 que agregan las reservas), se requiere únicamente del sitio 2 y del contingente de ambas reservas para contar con $80 \%$ de las especies (Cuadro 5). Nuevamente Manantlán desempeña un papel preponderante al proteger $68.3 \%$ de los elementos endémicos, con un complemento modesto de la reserva El Jabalí (5 especies).

El ejercicio realizado con los valores de rareza requirió, además de las dos reservas incluidas en el análisis, de un sitio más para incorporar $75 \%$ de las especies y su resultado es similar al obtenido con la riqueza total (Cuadro 5). Al igual que los casos anteriores, las reservas contribuyen con más de 65\% del objetivo de conservación (Cuadro 5). Únicamente los sitios 9 y 23 fueron seleccionados consistentemente en los tres algoritmos usados. 
Cuadro 5. Prioridades de conservación para la flora leñosa del estado de Colima con base en tres algoritmos de selección, tomando en cuenta la presencia de las especies en los 24 sitios censados y en las reservas de Colima (RBM, Reserva de la Biosfera Manantlán; APJ, Área de Protección Forestal y Refugio de la Fauna Silvestre El Jabalí). En cada prioridad se indica el sitio seleccionado, el número de especies que conserva (total y especies endémicas) y el valor de rareza. El porcentaje del total acumulado por localidad para los distintos algoritmos se muestra entre paréntesis.

\begin{tabular}{|c|c|c|c|c|c|c|}
\hline \multirow[t]{2}{*}{ Prioridad } & \multicolumn{2}{|c|}{ Riqueza total } & \multicolumn{2}{|c|}{ Riqueza endémicas } & \multicolumn{2}{|c|}{ Valor de rareza } \\
\hline & Sitio & $\begin{array}{l}\text { Especies } \\
(\mathrm{N}=552)\end{array}$ & Sitio & $\begin{array}{l}\text { Especies } \\
(\mathrm{N}=120)\end{array}$ & Sitio & $\begin{array}{c}\text { Especies } \\
(\mathrm{N}=552)\end{array}$ \\
\hline 1 & RBM & $295(53.4)$ & RBM & $82(68.3)$ & RBM & $208.8(55.3)$ \\
\hline 2 & APJ & $77(67.4)$ & APJ & $5(72.5)$ & APJ & $68.9(73.5)$ \\
\hline 3 & 11 & $36(73.9)$ & 2 & $11(80.0)$ & 9 & $13.8(77.2)$ \\
\hline 4 & 5 & $22(77.9)$ & 23 & $8(86.7)$ & 5 & $10.7(80.0)$ \\
\hline 5 & 15 & $16(80.8)$ & 8 & $4(90.0)$ & 24 & $7.8(82.1)$ \\
\hline 6 & 9 & $13(83.2)$ & 9 & $3(92.5)$ & 10 & $7.2(84.0)$ \\
\hline 7 & 23 & $13(85.5)$ & 12 & $2(94.2)$ & 16 & $6.4(85.7)$ \\
\hline 8 & 3 & $10(87.3)$ & 10 & $2(95.8)$ & 23 & $6.2(87.3)$ \\
\hline
\end{tabular}

La figura 2A muestra la disposición geográfica de los sitios que resultaron más importantes en los dos tipos de análisis de este trabajo (muestreo e inclusión de reservas) y se observa que tanto el 9 como el 19 se encuentran en el territorio de las reservas naturales. De esta manera cobra especial significancia la protección de las localidades 5, 11 y 23, al no ubicarse dentro de alguna de las áreas de protección de Colima. Otro sitio que convendría destacar es el 2, ya que ocupa posiciones relevantes respecto a su endemismo en los ejercicios con o sin reservas (Cuadros 4 y 5). Las UA en las que se distribuyen estos cuatro sitios abarcan en conjunto 16.6\% (919.6 $\mathrm{km}^{2}$ ) de la superficie estatal.

\section{DISCUSIÓN}

El uso de los gradisectos permitió obtener un listado de 355 especies de árboles (256 determinadas a nivel de especie) y 62 de lianas (41 especies), durante aproximadamente seis meses de trabajo, lo que incluye el tiempo de muestreo y 
las labores de determinación del material recolectado. Si se consideran únicamente los taxones determinados a nivel de especie, los gradisectos permitieron cuantificar $46.5 \%$ de las 550 especies arbóreas registradas por Padilla-Velarde et al. (2006) para Colima, cuyo trabajo requirió realizar colectas de campo intensivas durante un año y la revisión de ejemplares de herbario colectados previamente durante varios años de exploración botánica. El porcentaje de endemismo fue cercano entre ambos estudios, ya que para todo el estado se registra un valor de $43.4 \%$, mientras que en el presente estudio fue de $29.2 \%$.

Además de la menor demanda de recursos económicos, de tiempo y personal especializado invertidos en cuantificar un alto número de especies árboles y lianas, una ventaja adicional de este muestreo es que permite asociar una determinada composición de plantas a cada una de las variables utilizadas en la estratificación ambiental (geomorfología, altitud y tipo de vegetación) y en caso de interés futuro realizar análisis predictivos sobre la distribución de taxones particulares (Guisan y Thuiller, 2005; Sánchez-Cordero et al., 2005; Pawar et al., 2007; Téllez et al., 2010), para hacer inferencias sobre su estado de conservación en el estado.

Por otro lado, la utilización de UA en evaluaciones de diversidad biológica es un asunto controversial, ya que su definición depende de la precisión de las variables que se usan para delimitarlas (Margules y Redhead, 1995; Gaston, 1996). Sin embargo, su uso se ha justificado en aras de mejorar la eficiencia de los recursos para el diseño de muestreos a nivel de paisaje (Kirkpatrick y Brown, 1994; Jayasuriya y Abayawaradana, 2008). Otro aspecto que se discute para las UA es que una mayor estratificación ambiental puede generar un esquema de áreas de conservación mucho más definido. Si bien se puede estar de acuerdo con la afirmación anterior, agregar más variables ambientales incrementa el número de UA que se pueden reconocer en una región y, por lo tanto, los recursos necesarios para implementar un muestreo representativo. Para el caso específico de Colima, por ejemplo, al sumar otra variable ambiental a las empleadas en el presente estudio (e. g. tipo de suelo con 16 clases), el número de UA se eleva de 53 a 241, haciendo poco práctica la cuantificación de su riqueza vegetal.

También se puede cuestionar la representatividad de los resultados al no inventariar todas las UA que se delimitaron para Colima, ya que éstas podrían contener una alta riqueza de especies o de endemismos. La exclusión de estas unidades fue justificada, ya que debido a su inaccesibilidad, su muestreo hubiera incrementado los costos económicos del proyecto y los recursos humanos calificados que se requieren para esta tarea. Otra razón adicional para desestimar su estudio es que sólo representan $8.4 \%$ del estado de Colima y estas pequeñas superficies son más 
vulnerables a sufrir efectos de borde o tienen una mayor probabilidad de ser eliminadas por perturbaciones naturales o antropogénicas.

El tema central de este trabajo es la ubicación y orden de las prioridades de conservación. La importancia de los sitios 5, 9, 11, 19 y 23 es notable, ya que se ubican entre las seis primeras opciones en tres de los cuatro algoritmos de selección empleados (Cuadro 4). En este sentido destaca el alto grado de coincidencia entre las áreas más ricas en especies y las que presentan un elevado grado de endemismo, un hallazgo que también ha sido documentado para otros grupos de plantas en México (García-Mendoza, 1995; Villaseñor e Ibarra-Manríquez, 1998; Ibarra-Manríquez et al., 2002; Lira et al., 2002; Villaseñor et al., 2003). No obstante, es necesario recordar que los resultados obtenidos con inventarios de vertebrados muestran una tendencia opuesta, lo que hace que una selección de áreas prioritarias para estos grupos sea más complicada de alcanzar (Flores-Villela, 1993; Escalante-Pliego et al., 1993; Fa y Morales, 1993; Peterson et al., 1993; Prendergast et al., 1993).

La inclusión de las áreas de protección previamente establecidas en los ejercicios de priorización de sitios para la conservación se ha considerado pertinente para maximar la complementariedad en los atributos a preservar y evitar su redundancia cuando los recursos dedicados al respecto, como usualmente sucede, son limitados (Rebelo, 1994; Margules et al.,1994; Barnard et al., 1998; Margules y Pressey, 2000; Ceballos, 2007; Pinto y Grelle, 2009). Al realizar este tipo de ejercicio para Colima se revela que la aportación de las reservas para la protección de su flora leñosa es sumamente importante, ya que albergan $67.4 \%$ de la riqueza, $72.5 \%$ del endemismo y $73.5 \%$ del índice de valor de rareza de las especies (Cuadro 5), sobre todo si se considera que ocupan únicamente $2.7 \%$ del territorio del estado. Aunado a lo anterior, ocho especies en tres distintas categorías de amenaza de la NOM-059 (Anónimo, 2010) se registran para Manantlán: I) amenazadas (Albizia occidentalis Brandegee, Astronium graveolens Jacq., Guaiacum coulteri A. Gray, Sideroxylon capiri (A. DC.) Pittier y Tabebuia chrysantha G. Nicholson), II) en peligro de extinción (Litsea glaucescens Kunth) y III) sujetas a protección especial (Saurauia serrata DC. y Cnidoscolus autlanensis Breckon). El establecimiento de esta reserva, a pesar de basarse inicialmente en la protección de una "Especie Bandera" (Zea diploperennis H.H. Iltis, Doebley \& R. Guzmán, Poaceae), ha sido favorable para la conservación de un alto número de plantas de Colima, particularmente de elementos endémicos a México. También destaca el hecho de que Manantlán cuenta con una estrategia que concilia la conservación y el manejo de recursos (Jardel et al., 2006), lo que le ha permitido desempeñar un papel efectivo en la protección de la biodiversidad (Ceba1los, 2007; Figueroa y Sánchez-Cordero, 2008). 
Los sitios 5, 9 y 23 son los más complementarios a la riqueza que albergan las reservas de Colima, los cuales coinciden en las seis primeras prioridades en al menos dos de los algoritmos usados (Cuadro 5), aunque es importantte destacar que el 9 queda dentro del Parque Nacional Volcán de Colima. En conjunto con los sitios 5 y 23 , sería interesante considerar el 2, ya que es el más complementario en su componente endémico al que se localiza en las reservas (Cuadro 5) y el que ocupó la cuarta posición al respecto en el ejercicio que no consideró a éstas (Cuadro 4). Una situación similar se observa con el sitio 11, el primero con mayor riqueza después de Manantlán y El Jabalí (Cuadro 5) y en donde se registró el número más alto de especies de los sitios muestreados (Cuadro 3).

En conclusión, las UA donde se localizan los sitios 2, 5, 11 y 23 resultan ser las de mayor importancia en esquemas futuros de conservación de Colima, porque su ubicación está fuera de las actuales áreas de protección (Fig. 2). Debido a que la distribución de estas UA no es continúa, establecer una nueva reserva no sería sencillo. Sin embargo, una opción viable sería contar con un sistema de corredores protegidos con vegetación nativa conservada entre las UA donde se localizan los sitios más cercanos ( 2,5 y 11), las que incluso podrían eventualmente conectarse con la porción sureña de la reserva de Manantlán (Fig. 2B), aminorando los efectos de la falta de continuidad de los hábitats.

Adicionalmente, en la propuesta de áreas de conservación sería igualmente recomendable agregar tipos de vegetación, que si bien no sobresalen por su riqueza arbórea, son valiosos por la notable diversidad que albergan de otras formas de crecimiento vegetal (e. g. las hierbas) como es el caso de los bosques de Pinus-Quercus (UA 4 o 14) o bien los manglares (UA 13). Estos últimos, son comunidades especialmente amenazadas por los cambios de uso de suelo en Colima y que además de contener especies en la categoría de amenaza sujetas a protección especial (Avicennia germinans (L.) L., Conocarpus erectus L., Laguncularia racemosa (L.) C.F. Gaertn. y Rhizophora mangle L., Anónimo, 2010), son reconocidos a nivel mundial por ser un enlace de importantes procesos de flujo de energía entre los ecosistemas terrestres y los estuarinos costeros (Lugo y Snedaker, 1974).

Se espera que los resultados de esta investigación fomenten el interés por caracterizar la distribución de otros grupos de plantas y animales en la zona de estudio y promuevan la gestión de nuevas áreas de protección tomando en cuenta aspectos económicos, políticos y socialmente aceptables, que les aseguren una viabilidad a largo plazo. Este complejo ejercicio es urgente de realizar si se desean preservar muestras representativas de la biodiversidad de los árboles y las lianas de Colima. 
Martínez-Cruz e Ibarra-Manríquez: Áreas prioritarias de conservación para la flora leñosa de Colima

\section{AGRADECIMIENTOS}

Al Consejo Nacional de Ciencia y Tecnología (CONACyT) por el financiamiento del trabajo de campo (Proyecto 33067-V). Este estudio formó parte de la tesis de maestría del primer autor, desarollada en el Posgrado de Ciencias Biólogicas de la Universidad Nacional Autónoma de México, para la cual contó con una beca crédito del Consejo Nacional de Ciencia y Tecnología. Se agradece la colaboración en el trabajo de campo de Abraham Ramos López, Libertad Arredondo Amezcua, Miguel Ángel Salinas Melgoza, María de las Nieves Barranco León, Ana Guadalupe Rocha Loredo, Ana Patricia del Castillo Batista y Roberto Carlos Sáyago Lorenzana. Diversos problemas relacionados con el SIG fueron resueltos con la ayuda del Dr. Jesús Fuentes Junco, mientras que los relacionados con el manejo de bases de datos fueron resueltos por el MTI. Heberto Ferreira Medina y el Ing. Alberto Valencia García. También se reconoce la intervención del Dr. Gerardo Bocco Verdinelli y del M. en C. Luis M. Morales M. para obtener los archivos sobre el modelo digital de elevación de Colima. Por úlitmo se agradece a la M. en C. Leonor Solís Rojas por su ayuda en la vectorización de los gráficos de este manuscrito.

\section{LITERATURA CONSULTADA}

Anónimo. 1981. Síntesis geográfica de Colima. Coordinación General de Estadística Geografía e Informática, Secretaría de Programación y Presupuesto. México, D.F., México. $31 \mathrm{pp}$.

Anónimo. 1983. Carta edafológica 1:250 000 E13-4-5, E13-6 y E13-6-9. Instituto Nacional de Estadística Geografía e Informática, México, D.F., México.

Anónimo. 1985. Red de carreteras. Escala 1:1000 000. Digital Chart of the World. México.

Anónimo. 2001. Información geográfica por entidad federativa: Colima. Instituto Nacional de Estadística Geografía e Informática. http://www.inegi.gob.mx/entidades/espanol/ fcol.html (Consultado 25 octubre 2010).

Anónimo. 2010. Norma oficial mexicana NOM-059-ECOL-2010. Protección ambientalespecies nativas de México de flora y fauna silvestres- categorías de riesgo y especificaciones para su inclusión, exclusión o cambio. Lista de especies en riesgo. Diario Oficial de la Federación, 2a. Sección. 30 de diciembre de 2010. México, D.F., México. pp. 1-78.

Austin, M. P. y P. C. Heyligers. 1991. New approach to vegetation survey design: gradsect sampling. In: Margules, C. R. y M. P. Austin (eds.). Nature conservation: costeffective biological surveys and data analysis. Commonwealth Scientific and Industrial Research Organization. Canberra, Australia. pp. 31-36. 
Barnard, P., C. J. Brown, A. M. Jarvis, A. Robertson y L. van Rooyen. 1998. Extending the Namibian protected area network to safeguard hotspots of endemism and diversity. Biodivers. Conserv. 7: 531-547.

Caldecott, J. O., M. D. Jenkins, T. H. Johnson y B. Groombridge. 1996. Priorities for conserving global species richness and endemism. Biodivers. Conserv. 5: 699-727.

Cardoso, P., I. Silva, N. G. de Oliveira y A. Serrano. 2004. Indicator taxa of spider (Araneae) diversity and their efficiency in conservation. Biol. Conserv. 120: 517-524.

Ceballos, G. 2007. Conservation priorities for mammals in megadiverse Mexico: the efficiency of reserve networks. Ecol. Appl. 17: 569-578.

Cuevas, R., N. M. Núñez-López, L. Guzmán-Hernández y F. J. Santana-Michel. 1998. El bosque tropical caducifolio en la reserva de la Biosfera Sierra de Manantlán, JaliscoColima, México. Bol. Bot. Univ. Guad. 5: 445-491.

Dávila, P. y V. Sosa. 1994. El conocimiento florístico de México. Bol. Soc. Bot. Méx. 55: 21-27.

Di Castri, F., J. R. Vernhes y T. Younès. 1992. Inventorying and monitoring biodiversity: a proposal for an international network. Biol. Int. Special Issue 27: 1-28.

Escalante-Pliego, P., A. G. Navarro y A. T. Peterson. 1993. A geographic, ecological, and historical analysis of land bird diversity in Mexico. In: Ramamoorthy, T. P. (ed.). Biological diversity of Mexico: origins and distribution. Oxford University Press. New York, USA. pp. 279-304.

Fa, J. E. y L. M. Morales. 1993. Patterns of mammals diversity in Mexico. In: Ramamoorthy, T. P. (ed.). Biological diversity of Mexico: origins and distribution. Oxford University Press. New York, USA. pp. 315-352.

Finch, O. D. y J. Löffler. 2010. Indicators of species richness at the local scale in an alpine region: a comparative approach between plant and invertebrate taxa. Biodivers. Conserv. 19: 1341-1352.

Figueroa, F. y V. Sánchez-Cordero. 2008. Effectiveness of natural protected areas to prevent land use and land cover change in Mexico. Biodivers. Conserv. 17: 3223-3240.

Fleishman, E., J. R. Thomson, R. Mac Nally, D. D. Murphy y J. P. Fay. 2005. Using indicator species to predict species richness of multiple taxonomic groups. Conserv. Biol. 19: 1125-1137.

Flores-Villela, O. 1993. Herpetofauna of Mexico: distribution and endemism. In: Ramamoorthy, T. P. (ed.). Biological diversity of Mexico: origins and distribution. Oxford University Press. New York, USA. pp. 251-278.

Flores-Villela, O. y P. Gerez. 1994. Biodiversidad y conservación en México: vertebrados, vegetación y uso del suelo. Comisión Nacional para el Conocimiento y Uso de la Biodiversidad. México, D.F. 439 pp.

García, E. 1981. Modificaciones al sistema de clasificación climática de Koeppen. Instituto de Geografía, Universidad Nacional Autónoma de México. México, D.F. $252 \mathrm{pp}$.

García-Mendoza, A. 1995. Riqueza y endemismos de la familia Agavaceae en México. In: Linares, E., P. Dávila, F. Chiang, R. Bye y T. Elias (eds.). Conservación de plantas en peligro de extinción: diferentes enfoques. Instituto de Biología, Universidad Nacional Autónoma de México. México, D.F., México. pp. 51-75. 
Martínez-Cruz e Ibarra-Manríquez: Áreas prioritarias de conservación para la flora leñosa de Colima

Gaston, K. J. 1996. Species richness: measure and measurement. In: Gaston, K. J. (ed.). Biodiversity: A biology of numbers and difference. Blackwell Science Ltd. Oxford, UK. pp. 77-113.

Gentry, A. 1982. Patterns of neotropical plant species diversity. In: Hecht, M. K., B. Wallace y G. T. Prance (eds.). Evolutionary biology. Plenum Press. New York, USA. pp. $1-84$.

Gillison, A. N. 1983. Gradient oriented sampling for resource surveys: the gradsect method. In: Myers, K., C. R. Margules e I. Musto (eds.). Survey methods for nature conservation. Commonwealth Scientific and Industrial Research Organisation. Canberra, Australia. pp. 349-374.

Guisan, A. y W. Thuiller. 2005. Predicting species distribution: offering more than simple habitat models. Ecol. Letters 8: 993-1009.

Halffter, G. y M. E. Favila. 1993. The Scarabaeinae (Insecta: Coleoptera): an animal group for analyzing, inventorying and monitoring biodiversity in tropical rainforests and modified landscapes. Biol. Int. 27: 15-21.

Ibarra-Manríquez, G., J. L. Villaseñor, R. Durán y J. Meave. 2002. Biogeographical analysis of the tree flora of the Yucatan Peninsula. J. Biogeo. 29: 17-29.

Jardel, P. E. J., E. Santana C. y S. H. Graf M. 2006. Investigación científica y manejo de la Reserva de la Biosfera Sierra de Manantlán. In: Oyama, K. y A. Castillo (coord.). Manejo, conservación y restauración de recursos naturales de México. Perspectivas desde la investigación científica. Universidad Nacional Autónoma de México y Siglo XXI editores. México, D.F., México. pp. 127-153.

Jayasuriya, A. H. M. y S. D. Abayawardana. 2008. A study to determinate the changes in the biodiversity values of southern Sinharaja and Kanneliya forest after the implementation of GEF medium sized project. Global Environmental Facility and United Nations Development Programme. Colombo, Sri Lanka. 67 pp.

Kerr, T. J. 1997. Species richness, endemism and the choice of areas for conservation. Conserv. Biol. 11: 1094-1100.

Kirkpatrick, J. B. y M. J. Brown. 1994. A comparison of direct and environmental domain approaches to planning reservation of forest higher plant communities and species in Tasmania. Conserv. Biol. 8: 217-224.

Lira, R., J. L. Villaseñor y E. Ortiz. 2002. A proposal for the conservation of the family Cucurbitaceae in Mexico. Biodivers. Conserv. 11: 1699-1720.

Lugo A. E. y S. C. Snedaker. 1974. The ecology of mangroves. Ann. Rev. Ecol. Syst. 5: 39-64.

Margules, C. R. y M. B. Usher. 1981. Criteria used in assessing wildlife conservation potential: a review. Biol. Conserv. 21: 79-109.

Margules, C. R., A. O. Nicholls y R. L. Pressey. 1988. Selecting networks of reserves to maximize biological diversity. Biol. Conserv. 43: 63-76.

Margules, C. R., I. D. Cresswell y A. O. Nicholls. 1994. A scientific basis for establishing networks of protected areas. In: Forey, L., C. J. Humphries y R. I. Vane-Wright (eds.). Systematic and conservation evaluation. Clarendon Press. Oxford, UK. pp. 327-350

Margules, C. R. y T. D. Redhead. 1995. Guidelines for using the BioRap methodology and tools. Commonwealth Scientific and Industrial Research Organisation. Canberra, Australia. 70 pp. 
Margules, C. R. y R. L. Pressey. 2000. Systematic conservation planning. Nature 405: 243253.

Margules, C. R., R. L. Pressey y P. H. Williams. 2002. Representing biodiversity: data and procedures for identifying priority areas for conservation. J. Bioscience 27: 309326.

Myers, N., R. A. Mittermeier, C. G. Mittermeier, G. A. da Fonseca y J. Kent. 2000. Biodiversity hotspots for conservation priorities. Nature 403: 853-858.

Olson, D. M. y E. Dinerstein. 2002. The global 200: priority ecoregions for global conservation. Ann. Missouri Bot. Gard. 89: 199-224.

Padilla-Velarde, E., R. Cuevas-Guzmán, G. Ibarra-Manríquez y S. Moreno-Gómez. 2006. Riqueza y biogeografía de la flora arbórea del estado de Colima, México. Rev. Mex. Biodiv. 77: 271-295.

Padilla-Velarde, E., R. Cuevas-Guzmán y S. D. Koch. 2008. Plantas vasculares y vegetación de la parte alta del arroyo Agua Fría, municipio de Minatitlán, Colima, México. Act. Bot. Mex. 84: 25-72.

Palacio-Prieto, J. L., G. Bocco, A. Velázquez, J. F. Mas, F. Takaki-Takaki, A. Victoria, L. Luna-González, G. Gómez-Rodríguez, J. López-García, M. Palma, I. Trejo-Vázquez, A. Peralta, J. Prado-Molina, A. Rodríguez-Aguilar, R. Mayorga-Saucedo y F. González. 2000. La condición actual de los recursos forestales en México: resultados del Inventario Forestal Nacional 2000. Invest. Geogr. Bol. Inst. Geog. 43: 183-203.

Pawar, S., M. S. Koo, C. Kelley, M. F. Ahmed, S. Chaudhuri y S. Sarkar. 2007. Conservation assessment and prioritization of areas in Northeast India: priorities for amphibians and reptiles. Biol. Conserv. 136: 346-361.

Peterson, A. T., O. A. Flores-Villela, L. S. León-Paniagua, J. E. Llorente-Bousquets, M. A. Luis-Martínez, A. G. Navarro-Singüenza, M. G. Torres-Chávez e I. VargasFernández. 1993. Conservation priorities in Mexico: moving up in the world. Biodivers. Letters 1: 33-38.

Pinto, M. P. y C. E. V. Grelle. 2009. Reserve selection and persistence: complementing the existing Atlantic Forest reserve system. Biodivers. Conserv. 18: 957-968.

Prendergast, J. R., R. W. Quinn, J. H. Lawton, B. C. Eversham y D. W. Gibbons. 1993. Rare species, the coincidence of diversity hotspot and conservation strategies. Nature 365: 335-337.

Pressey, R. L., I. R. Johnson y P. D. Wilson. 1994. Shades of irreplaceability: towards a measure of the contribution of sites to a reservation goal. Biodivers. Conserv. 3: 242262.

Pressey, R. L. y K. H. Taffs. 2001. Sampling of land types by protected areas: three measures of effectiveness applied to western New South Wales. Biol. Conserv. 101: 105-117.

Raven, P. y E. O. Wilson. 1992. A fifty-year plan for biodiversity surveys. Science 258: 1099-1100.

Rebelo, A. G. 1994. Iterative selection procedures: centers of endemism and optimal placement of reserves. Strelitzia 1: 231-257.

Rothschild, B., E. J. Lott y A. Sanders. 1992. A report to the Fundación Ecológica Cuixmala on the floristic surveys of 1990-91 of the Cuixmala-Cumbres and El Jabalí Reserves in Mexico. Fundación Ecológica Cuixmala. México, D.F. 134 pp. 
Rzedowski, J. y R. McVaugh. 1966. La vegetación de Nueva Galicia. Contrib. Univ. Mich. Herb. 9: 1-123.

Rzedowski, J. y G. Calderón de Rzedowski. 1993. Bignoniaceae. Flora del Bajío y de Regiones Adyacentes 22: 1- 44.

Sánchez-Cordero, V., P. Illoldi-Rangel, M. Linaje, S. Sarkar y A. T. Peterson. 2005. Deforestation and extant distributions of Mexican endemic mammals. Biol. Conserv. 126: 465-473.

Schmit, J. P., G. M. Mueller, P. R. Leacock, J. L. Mata, Q. Wu y Y. Huang. 2005. Assessment of tree species richness as a surrogate for macrofungal species richness. Biol. Conserv. 121: 99-110.

Stork, N. E. 1994. Inventories of biodiversity: more than a question of numbers. In: Forey, P. L., C. J. Humphries y R. I. Vane-Wright. (eds.). Systematic and conservation evaluation. Clarendon Press. Oxford, UK. pp. 81-100.

Téllez, O., V. Farías, P. Dávila, J. L. Stein, R. Lira y F. Botello. 2010. Mammalian diversity in climatic domains for Tehuacán-Cuicatlán Biosphere Reserve, Mexico. Rev. Mex. Biodiv. 81: 863-874.

Verstappen, H. T. y R. A. van Zuidam. 1991. The ITC system of geomorphologic survey. International Institute for Aerospace Survey and Earth Sciences. Enschede. Holanda. $89 \mathrm{pp}$.

Villaseñor, J. L. y G. Ibarra-Manríquez. 1998. La riqueza arbórea de México. Bol. Inst. Bot. Univ. Guad. 5: 95-105.

Villaseñor, J. L., G. Ibarra-Manríquez, J. A. Meave y E. Ortiz. 2003. Biogeografía y conservación de los bosques tropicales húmedos de México. In: Llorente, J. y J. J. Morrone (eds.). Introducción a la biogeografía en Latinoamérica: teorías, conceptos, métodos y aplicaciones. Facultad de Ciencias, Universidad Nacional Autónoma de México. México, D.F., México. pp. 209-216.

Virolainen, K. M., P. Alroth, E. Hyvarinen, E. Korkeamaki, J. Mattila, J. Paivinen, T. Rintala, T. Suomi y J. Suhonen. 2000. Hot spots, indicator taxa, complementarity and optimal networks of taiga. Proc. Biol. Sci. 267: 1143-1147.

Wessels, K. J., A. S. Van Jaarsveld, J. D. Grimbeek y M. J. Van Der Linde. 1998. An evaluation of the gradsect biological survey method. Biodivers. Conserv. 7: 1093-1121. 